\title{
Constrictions Fabricated on YBCO Thin Film using the Femtosecond Laser: Limiting Factors
}

\author{
P.O. Umenne ${ }^{a}$ And V.V. SRINIVASU ${ }^{b, *}$ \\ ${ }^{a}$ Department of Electrical and Mining Engineering, University of South Africa, Johannesburg 1710, South Africa \\ ${ }^{b}$ Department of Physics, University of South Africa, Johannesburg 1710, South Africa
}

\begin{abstract}
In this paper we report on the development of a new application of the femtosecond laser, namely, the fabrication of micron, sub-micron and nano sized constrictions on YBCO superconductive thin films. The fabricated micron, sub-micron and nano constrictions have widths of $1.09 \mu \mathrm{m}, 705 \mathrm{~nm}$ and $394.8 \mathrm{~nm}$, respectively. The thickness of these constrictions ranged from about $160 \mathrm{~nm}$ to $190 \mathrm{~nm}$, as defined by the depth profile on the 3D AFM images. The length of these constrictions ranged from $7.2 \mu \mathrm{m}$ to $7.5 \mu \mathrm{m}$. The length of these constrictions depends on the size of the laser ablation spot used for cutting and on the separation distance between the laser ablation spots along the width of the sample.
\end{abstract}

DOI: 10.12693/APhysPolA.134.100

PACS/topics: femtosecond laser, nanostructures, atomic force microscope, Josephson junctions

\section{Introduction}

The femtosecond laser can be used in many applications such as micromachining of bulk glass [1], writing waveguides in glass [2], polymerization of inorganicorganic hybrid materials [3], as a high accuracy optical distance measuring tool [4], in the medical field for eye corneal refractive surgery [5], for the fabrication of temperature sensors [6], for colorizing metals [7], etc.

In this paper we report on a new application. We use the ablative properties of the femtosecond laser to fabricate constrictions on a superconductive YBCO thin film. These constrictions could later be used as Josephson junctions. Josephson junctions have many applications of their own, such as SQUID's magnetic sensors and as elements of qubit technology. In addition, the limiting factors for the fabrication of "nano" constrictions are discussed.

\section{Experimental details}

\subsection{Laser specifications and settings}

The employed femtosecond laser has a wavelength of $795 \mathrm{~nm}$ and a pulse duration time of $130 \mathrm{fs}$. The laser ablation width achieved using a spherical convex lens of $30 \mathrm{~mm}$ focal length was on average $15.53 \mu \mathrm{m}$. When the separation distance between the laser ablation spots $S_{\mathrm{W}}$ was set to $16.5 \mu \mathrm{m}$ the sub-micron sized constriction of $705 \mathrm{~nm}$ was produced. With a separation distance between the laser ablation spots $S_{\mathrm{W}}$ of $16.0 \mu \mathrm{m}$ we achieved the nano sized constriction of $394.8 \mathrm{~nm}$. Lastly with a separation distance of $17.0 \mu \mathrm{m}$ we achieve a micron sized

*corresponding author; e-mail: vallavs@unisa.ac.za constriction with a width of $1.09 \mu \mathrm{m}$. This dependence is summarized in Eq. (1);

constrict. width $=S_{\mathrm{W}}$ - laser ablation spot size. (1) The fabricated constrictions where S-shaped. With such shape it is possible to regulate both the width and the length of the constriction, as described elsewhere [8]. The width is controlled by bringing the laser ablation spots closer along the length of the thin film sample, while the length can be controlled by separating the laser ablation spots along the width of the thin film sample.

However, as the laser ablation spots are brought closer together along the length of the sample the constriction width tends to become smaller until in some instances the fabricated constrictions would collapse.

\section{Results and discussion}

\subsection{Factors that limit the fabrication of nanostructures}

When nano-structuring a material using the femtosecond laser, the width of the constriction or the diameter of the smallest structure that can be fabricated is determined by Eq. (2) below;

$$
d=k \lambda / \mathrm{NA} q^{1 / 2}
$$

where $d$ is the diameter of the constriction made or the width, $\lambda$ is the wavelength of the femtosecond laser, NA is the numerical aperture of an objective plano convex lens of the focusing optics, $q$ is the energy band gap of the material sample being used for nano structuring. This equation was obtained from [9]. The main limiting factors of nano-fabrication are discussed below.

1. One of the main limiting factors for the fabrication of nano bridges is in the specification of the lens being used for optimizing the size of the laser ablation spot during focusing of the laser beam. The laser ablation spot size has to be as small as possible before cutting the constriction on the sample. The conventional spherical 
convex lenses, such as those with a relative focal length, place a limit on the size of the laser ablation spot they can produce because of their reduced focusing power. The smaller the laser ablation spot size, the smaller the constriction that can be fabricated. This is because if a constriction of nano range has to be made, then the spot size has to be in the $1-5 \mu \mathrm{m}$ range, so that the laser ablation spots can be brought closer to one another without clipping the constriction bridge. The objective plano convex lenses with such numerical apertures NA as $0.25,0.40$, 0.65 are more suitable to produce smaller laser ablation spot diameters and hence smaller constrictions because they are able to converge the laser beam more. Hence they are able to produce smaller ablations spots.

2. The energy band gap of the material being used for nano-structuring, in this case the YBCO thin film, also plays a role. The materials with a higher energy band gap tend to have the capability of producing smaller constriction sizes. For example YBCO thin film has an out-of-plane energy band gap of $5 \mathrm{meV}$ [10] which allows it to produce nano structures somewhere in the limit of $400 \mathrm{~nm}$. This could be one of the reasons why when the constriction of nano size (394.8 nm) was produced, the walls of the constriction had a tendency to collapse after fabrication. Other factors that contribute towards the collapse of the constriction include how close the ablation spots are brought to each other, vibration and the temperature in the clean room while cutting.

3. The last limiting factor is the radiation wavelength of the laser being used. The femtosecond laser used for this experiment had a radiation wavelength $\lambda$ of $795 \mathrm{~nm}$. Essentially this places a diffraction limit on the light that is incident on the sample from the laser when cutting. This limit also reduces the chances of cutting constrictions with diameter less than $795 \mathrm{~nm}$ without collapsing the constriction.

\subsection{Nano constriction $(0-500 \mathrm{~nm})$}

The nano sized constriction was fabricated with the following laser settings; separation distance between the laser ablation spots $S_{\mathrm{W}}=16.0 \mu \mathrm{m}$, the laser ablation spot size of $15.53 \mu \mathrm{m}$, laser power setting $2.035 \mathrm{~mW}$ and in the optical set up a spherical convex lens of focal length of $30 \mathrm{~mm}$ was utilised. The constriction width was measured and was determined to be $394.8 \mathrm{~nm}$ using the amplitude line fit, as can be seen in Fig. 1b. The scan area in Fig. 1 was set to $20.2 \times 20.2 \mu \mathrm{m}^{2}$ using the AFM software [11], which enables one to see that the constriction is clipped and not geometrically continuous. Figure 1a shows the topography line fit and Fig. 1b shows the amplitude line fit used to determine the width of the constriction.

\subsection{Sub-micron sized constriction (500-1000 nm)}

For the sub-micron sized constriction the separation distance between laser ablation spots $S_{\mathrm{W}}$ was $16.5 \mu \mathrm{m}$. The laser ablation spot size was $15.82 \mu \mathrm{m}$. The fabricated constriction is geometrically continuous as can be seen in

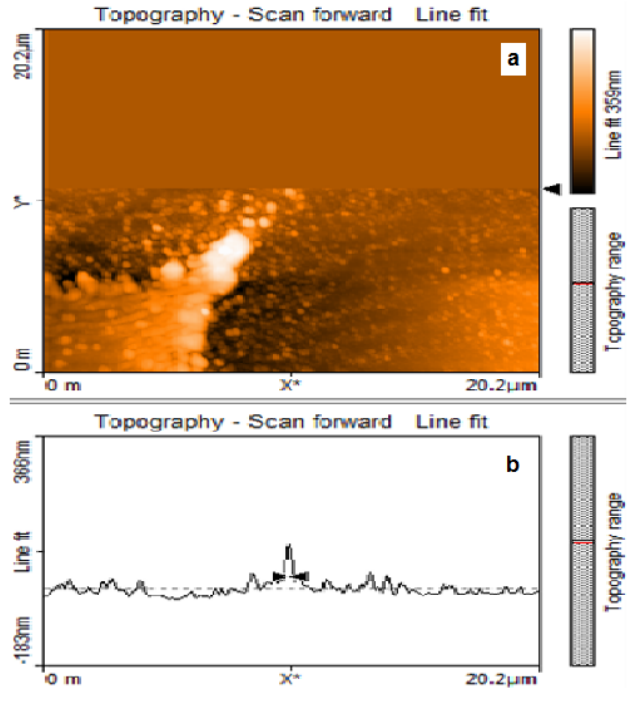

Fig. 1. AFM image of the nano constriction with the width of $394.8 \mathrm{~nm}$.

Fig. 2a. The scan area was set to $16.4 \times 16.4 \mu \mathrm{m}^{2}$ and the constriction width was measured to be $705 \mathrm{~nm}$ using the amplitude line fit of the constriction in Fig. $2 \mathrm{~b}$.

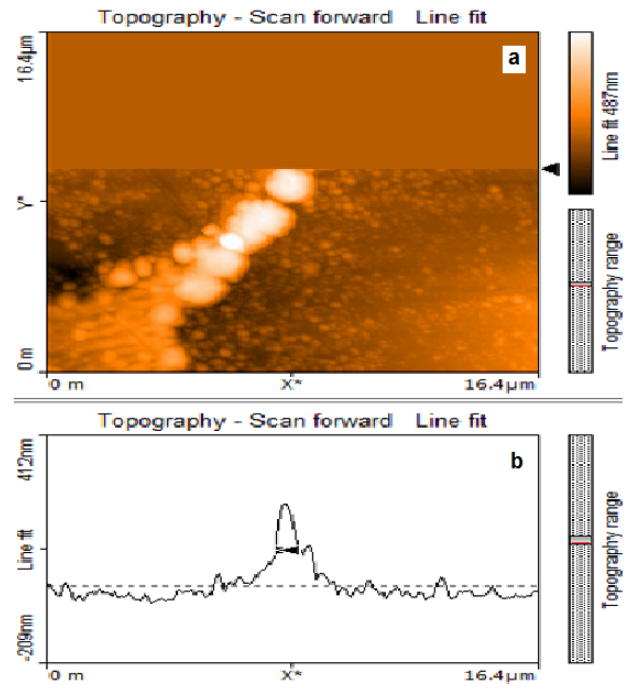

Fig. 2. AFM image of the sub-micron constriction with the width of $705 \mathrm{~nm}$ (shown by the black arrows in the profile image).

\subsection{Micron sized constriction (above $1000 \mathrm{~nm}$ )}

The micron sized constriction was fabricated with the separation distance between the laser ablation spots $S_{\mathrm{W}}=17.0 \mu \mathrm{m}$. The laser ablation spot size was $15.23 \mu \mathrm{m}$, which is slightly less than the ablation spot size for the nano constriction. The laser power was set to $2.0 \mathrm{~mW}$ and a spherical convex lens with a focal length 
of $30 \mathrm{~mm}$ was used. The constriction width was determined to be $1.09 \mu \mathrm{m}$, as can be seen from the amplitude line fit in Fig. 3b. The fabricated constrictions and their physical dimensions are summarized in Table I. Figure 3a shows the topography line fit of the constriction.

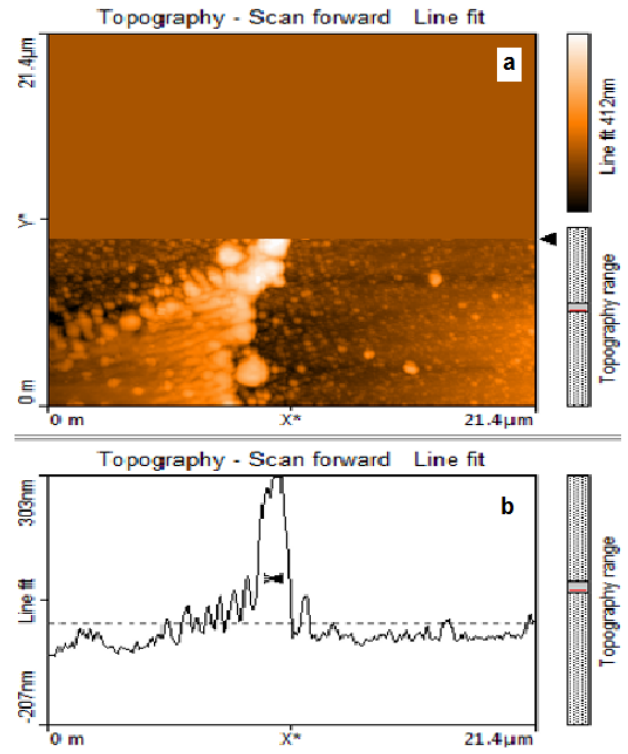

Fig. 3. AFM image of the micron constriction with the width of $1.09 \mu \mathrm{m}$.

TABLE I

Summary of prepared constrictions with all specifications.

\begin{tabular}{l|c|c|c}
\hline \hline Constriction type & Nano & Sub-micron & Micron \\
Number & 1 & 2 & 3 \\
$S_{\mathrm{W}}[\mu \mathrm{m}]$ & 16.0 & 16.5 & 17.0 \\
Qubit number on YBCO films & $3 \mathrm{rd}$ & $5 \mathrm{th}$ & $4 \mathrm{th}$ \\
Constriction width $[\mathrm{nm}]$ & 394.8 & 705 & 1090 \\
Ablation spot diameter $[\mu \mathrm{m}]$ & 15.53 & 15.8 & 15.23
\end{tabular}

\section{Conclusions}

We demonstrated a new application of femtosecond laser in fabrication of micron, sub-micron and nano sized constrictions that could be used as Josephson junctions. A $395 \mathrm{~nm}, 705 \mathrm{~nm}$ and $1.09 \mu \mathrm{m}$ wide constrictions where fabricated. The limitations of using the femtosecond laser as a nano structuring tool where discussed. The separation distances between the laser ablation spots $S_{\mathrm{W}}$ and the ablation spot sizes were determined experimentally for each constriction in order to be able to fabricate these constrictions.

\section{References}

[1] C.B. Schaffer, A. Brodeur, J.F. García, E. Mazur, Opt. Lett. 26, 93 (2001).

[2] K.M. Davis, K. Miura, N. Sugimoto, K. Hirao, Opt. Lett. 21, 1729 (1996).

[3] J. Serbin, A. Egbert, A. Ostendorf, B.N. Chichkov, R. Houbertz, G. Domann, J. Schulz, C. Cronauer, L. Fröhlich, M. Popall, Opt. Lett. 28, 301 (2003).

[4] K. Minoshima, H. Matsumoto, Appl. Opt. 39, 5512 (2000).

[5] A. Sugar, Curr. Opin. Ophthalmol. 13, 246 (2002).

[6] D. Grobnic, S.J. Mihailov, C.W. Smelser, H. Ding, IEEE Photonics Technol. Lett. 16, 2505 (2004).

[7] A.Y. Vorobyev, C. Guo, Appl. Phys. Lett. 92, 41914 (2008).

[8] P. Umenne, V.V. Srinivasu, Journal of Materials Science: Materials in Electronics 28, 5817 (2008).

[9] F. Korte, J. Serbin, J. Koch, A. Egbert, C. Fallnich, A. Ostendorf, B.N. Chichkov, Appl. Phys. A 77, 229 (2003).

[10] N. Hass, G. Deutscher, G. Desgardin, I. Monot, M. Weger, Journal of Superconductivity 5, 191 (1992).

[11] Nanosurf FlexAFM, Operating Instructions Version 3, NANOSURF 2011. 